\title{
INVERSÃO DA FORMA DE ONDA COMPLETA UTILIZANDO MÉTODOS DE OTIMIZAÇÃO LIVRES DE DERIVADAS
}

\author{
Rodrigo de Santana Santos $1^{1}$; Daniel E. Revelo $2^{1,2}$; Oscar M. Ladino $3^{1}$ \\ ${ }^{1} \mathrm{HPC} / \mathrm{SENAI-CIMATEC;} \mathrm{Salvador/BA;} \mathrm{e-mail:} \mathrm{rodrigo.santana@fieb.org.br}$ \\ ${ }^{2}$ CPGG/UFBA; Salvador/BA
}

\begin{abstract}
Resumo: A inversão da forma de onda (FWl: Full Waveform Inversion) é uma poderosa ferramenta na busca de modelos de velocidades de alta resolução. $\mathrm{Na}$ FWI convencional são empregados métodos determinísticos, o que exige uso de modelo inicial acurado para garantia de convergência. Neste trabalho apresentamos a FWI com emprego dos métodos heurístico: Optimização por Enxame de Partículas (PSO: Particle Swarm Optimization) e Algoritmo Genético (GA: Genetic Algorithm), nos chamados PSOFWI e GAFWI. Essas abordagens, são livres do cálculo de derivadas e permitem a obtenção do modelo de velocidades ótimo independentes da estimativa inicial. Os experimentos foram reunidos em duas seções: comparação da efetividade do GA e PSO na minimização de uma função analítica e a aplicação dos GAFWI e PSOFWI na inversão de velocidades 1D.
\end{abstract}

Palavras-Chave: GA; PSO; FWI; GAFWI; PSOFWI.

\section{FULL WAVEFORM INVERSION USING DERIVATIVE-FREE OPTIMIZATION METHOD}

\begin{abstract}
The Full Waveform Inversion method (FWI) It has excelled as powerful tool in the seek of the field velocity, due to its capacity to estimating models with high resolution. In a conventional fashion, the FWI by to use deterministic methods in the minimization process, need a good initial estimation. This work presents a nonconventional approach that uses nondeterministic methods of Genetic Algorithm (GA) and Particle Swarm Optimization (PSO) in the FWI scheme that is mentioned as GAFWI and PSOFWI methods. The work is divided into two phases: first, a performance comparison between GA and PSO to minimize analytical functions, and a second step with the application of GAFWI and PSOFWI in one-dimensional inversion.
\end{abstract}

Keywords: GA; PSO; FWI; GAFWI; PSOFWI. 


\section{INTRODUÇÃO}

$\mathrm{Na}$ busca do melhor entendimento da subsuperfície, a indústria do petróleo tem feito largo uso dos métodos geofísicos de exploração, em especial o sísmico, devido a sua capacidade de registrar reflexões associadas a refletores situados a alguns quilômetros de profundidade (i.e. [1]). O principal objetivo deste método é fornecer uma "imagem" da subsuperfície que permita inferir sobre as estruturas presentes na área de estudo. Dentre as principais técnicas de imageamento sísmico, a migração reversa no tempo (RTM, Reverse Time Migration) tem se destacado na geração de imagens de alta qualidade, tendo como pré-requisito a existência de um campo de velocidades com boa acurácia. Por essa razão, a obtenção do campo de velocidades tornou-se uma etapa altamente relevante no fluxo do processamento sísmico, o que justifica o enorme esforço que têm sido empregado no desenvolvimento de técnicas para a estimativa deste campo. Nesse contexto, a FWI tem ganhado cada vez mais importância, por sua capacidade de obter campos de velocidades com alta resolução. Em resumo, a FWI é uma técnica de otimização, que parte do dado observado e um modelo inicial, e busca obter um modelo de velocidades, tal que o dado modelado sobre este, apresente uma distância mínima do dado observado. O processo geralmente é realizado por um método determinísticos de inversão (p. ex., conjugado gradiente) e estes tipos de métodos convergem rapidamente para o mínimo mais próximo da estimativa inicial, no entanto, a sua convergência para um modelo ótimo depende da qualidade da estimativa inicial. Além disso, os métodos determinísticos exigem o cálculo de derivadas, o que geralmente é computacionalmente custoso. Buscando-se fugir das limitações presentes na FWI convencional, neste trabalho apresentamos a técnica FWI baseada nos algoritmos globais GA e PSO, resultando nos métodos denominados GAFWI e PSOFWI. Estes métodos, ao contrario dos métodos gradientes, não calculam derivadas no processo de inversão, e não guardam dependência entre os modelos estimado e inicial, podendo obter um modelo ótimo, mesmo partindo de um modelo ruim. As duas principais desvantagens dos métodos GAFWI e PSOFWI são: i) precisam de muitas avaliações do modelo para convergir, exigindo assim um número elevado de modelagens por diferenças finitas, tornando a sua aplicação computacionalmente cara; ii) e o fato desses métodos que serem afetados pelo número de parâmetros a inverter. A primeira limitação citada pode ser desprezada a depender do poder computacional presente. Quanto à segunda limitação, existem pesquisas que visam redimensionar o campo de velocidades, reduzindo assim o número de dimensões envolvidas no esquema de inversão (i.e. [2]). Neste trabalho fugimos das limitações por usar computadores poderosas e por aplicar as técnicas GAFWI e PSOFWI na estimativa de modelos de velocidades 1D.

\section{TEORIA DA INVERSÃO E MÉTODOS GAFWI E PSOFWI}

Quando se busca relacionar as propriedades ou parâmetros físicos de um modelo, $\boldsymbol{m}$ (p. ex., resistividade ou velocidade), a dados de um levantamento ou modelagem geofísica, $\boldsymbol{d}$, estamos buscando resolver um problema direto ou inverso. 
De maneira geral, os processos de modelagem e inversão distinguem-se quanto ao papel das variáveis $\boldsymbol{d}$ e $\boldsymbol{m}$. No problema direto, $\boldsymbol{m}$ representa a variável independente e $\boldsymbol{d}$ a dependente, no problema inverso os papéis são trocados. Isto é, quando os parâmetros $\boldsymbol{m}$ são conhecidos e deseja-se obter $\boldsymbol{d}$, estamos resolvendo o problema direto, ou simplesmente realizando uma modelagem geofísica. Obter $\boldsymbol{m}$ quando $\boldsymbol{d}$ é conhecido significa resolver um problema inverso. Em ambas as situações, sempre existe um modelo matemático $\boldsymbol{d}=\mathbf{G}(\boldsymbol{m})$ que relaciona ditas quantidades. No caso da FWI, $\boldsymbol{d}$ representa os traços do sismograma (dado sísmico), $\boldsymbol{G}$ é o operador de modelagem com base na equação da onda e $\boldsymbol{m}$ é o modelo de velocidades. Algumas vezes é possível encontrar um operador de inversão $\boldsymbol{G}^{-1}$, o qual aplicado em $\boldsymbol{d}$ resulta em $\boldsymbol{m}$, porém na $\mathrm{FWI}$ isso não é possível. Assim, a FWI é tratada como um problema de otimização da função objetivo $\mathbf{Q}(\mathbf{m})$, em que, uma estimativa inicial $\boldsymbol{m}_{\boldsymbol{0}}$, é melhorada iterativamente por:

$$
m_{k+1}=m_{k}+\Delta_{m k}
$$

onde, a cada iteração, o modelo $\boldsymbol{m}_{k}$ é atualizado para $\boldsymbol{m}_{k+1}$ por meio da pertubação $\Delta_{m k}$. Nos problemas clássicos da FWI, o termo $\Delta_{m k}$ é calculado diretamente por meio de $\boldsymbol{Q}\left(\boldsymbol{m}_{\boldsymbol{k}}\right)$, porém aqui propõe-se usar os métodos GA e PSO para este fim, dando origem aos métodos GAFWI e PSOFWI.

\subsection{Função Objetivo e Equação da Onda}

O processo de inversão baseado em otimização tem como princípio minimizar (ou maximizar) uma função objetivo $Q(m)$, que é definida como:

$$
\boldsymbol{Q}(\boldsymbol{m})=\left\|\boldsymbol{d}_{\boldsymbol{o b s}}-\boldsymbol{d}_{\boldsymbol{c a l}}\right\|_{2}
$$

onde, $\boldsymbol{d}_{\text {obs }}$ e $\boldsymbol{d}_{\text {cal }}$ representam os dados observados e modelados. No problema da $\mathrm{FWI}, \boldsymbol{d}_{\text {cal }}$ é obtido pela solução da equação da onda (3), onde $\mathbf{m}$ representa 0 modelo de velocidades corrente. A solução da a equação (3) é obtida numericamente usando o esquema de diferenças finitas (i.e. [3]).

$$
\frac{\partial^{2} \boldsymbol{P}(\mathbf{z}, \boldsymbol{t})}{\partial \mathbf{z}^{2}}=\frac{1}{\boldsymbol{m}^{2}} \frac{\partial^{2} \boldsymbol{P}(\mathbf{z}, \boldsymbol{t})}{\partial \boldsymbol{t}^{2}}
$$

onde, $\boldsymbol{P ( z , t )}$ é o campo de pressão, $\boldsymbol{z}$ a variável espacial e $\boldsymbol{t}$ representa o tempo, sendo $\mathbf{m}$ o perfil de velocidade representado pelo vetor: $\boldsymbol{m}=\left[m\left(z_{1}\right), m\left(z_{2}\right), \ldots, m\left(z_{n}\right)\right]$.

\subsection{Algoritmo Genético}

De acordo com a equação (2), a cada iteração, o modelo atual $\boldsymbol{m}_{\boldsymbol{k}}$ sofre uma atualização tornando-se $\boldsymbol{m}_{k+1}$, a qual pode ser estimada mediante o emprego do Algoritmo Genético (GA). O modelo $\boldsymbol{m}_{\boldsymbol{k}}$ que é recebido pelo GA, a cada iteração, é atualizado seguindo os princípios da evolução biológica de um sistema natural (população), onde os seres (modelos) mais adaptados a um dado ecossistema tendem a sobreviver e propagar suas características. No GA, a cada iteração, existe uma população de modelos candidatos a solução do problema (população), e sobre 
esses são realizados operações que simulam processos biológicos (seleção, cruzamento e mutação), gerando assim uma nova população. Espera-se que em um número finito de gerações a população de modelos convirja para a solução ótima do problema. Os GAs diferem dos métodos de inversão determinísticos nos seguintes aspectos: trabalham com um conjunto de parâmetros a ser otimizado de uma forma codificada, podendo ser binária por analogia aos cromossomos (i.e. [4]); usam diretamente a informação a ser otimizada (função objetivo), não utilizam derivadas ou outros conhecimentos auxiliares; utilizam regras de transição probabilística e não regras determinísticas, e isso evita a exigência de convergência local. O uso do GA envolve algumas etapas (chamadas de operadores genéticos) sendo as principais:

1. Geração: A população é gerada com base em um modelo inicial $\boldsymbol{m}_{\boldsymbol{0}}$.

2. Codificação: Os parâmetros são codificados de forma binária ou real (i.e. [2]). Na codificação binária, cada parâmetro pode ser representado por uma cadeia de bit semelhante a uma cadeia de DNA. Nesse tipo de codificação o custo de memória é elevado, o que torna a codificação real mais indicada em problemas de muitos parâmetros, como os de FWI.

3. Seleção: Seleciona-se os modelos mais aptos ao problema.

4. Cruzamento: Combina-se os modelos selecionados gerando novos.

5. Mutação: Etapa que insere aleatoriamente modificações em membros da população, mantendo a diversidade na população e evitando convergência prematura. O processo retorna sempre ao passo 3 até alcançar um critério.

\subsection{Otimização por Enxame de Partículas}

O PSO é um algoritmo evolutivo de otimização global inspirado no comportamento social de organismos biológicos de trabalhar em conjunto para localizar boas regiões com fontes de alimento, tais como bandos de pássaros ou peixes (i.e. [5]). Semelhante ao GA, é criado um enxame de modelos (ou partículas), e cada partícula $\left(\left\{\boldsymbol{m}_{i}\right\}^{p}{ }_{i=1}\right)$ se movimenta no espaço de modelos com velocidade $\left(\left\{\boldsymbol{v}_{i}\right\}^{p_{i=1}}\right)$. A velocidade da partícula $p$ depende de três fatores: a velocidade $p$ atual de cada partícula $\left(\left\{v_{i}(k)\right\}_{i=1}^{p}\right)$ (fator de inércia), a sua melhor posição ao longo de todo 0 processo até o momento, definida por $\left\{g_{i}\right\}^{P_{i=1}}$ (fator cognitivo) e a posição da melhor partícula de todo o enxame ao longo do processo, definida por $G$ (fator social). No início do PSO, as partículas são inicializadas em posições aleatórias no espaço de busca segundo uma distribuição de probabilidade uniforme. Em seguida, a cada iteração $k$, as velocidades $\left(\boldsymbol{V}_{i}\right)$ e as posições $\left(\boldsymbol{m}_{i}\right)$ das partículas são atualizadas por:

$$
\begin{aligned}
& \boldsymbol{v}_{\boldsymbol{i}}(\boldsymbol{k}+1)=\boldsymbol{\phi} \times\left(\boldsymbol{v}_{\boldsymbol{i}}(\boldsymbol{k})+\boldsymbol{a}_{1} \times \boldsymbol{a}_{l o c} \times\left(\boldsymbol{g}_{\boldsymbol{i}}-\boldsymbol{m}_{\boldsymbol{i}}(\boldsymbol{k})\right)+\boldsymbol{a}_{2} \times \boldsymbol{a}_{g l o b} \times\left(\boldsymbol{G}-\boldsymbol{m}_{\boldsymbol{i}}(\boldsymbol{k})\right)\right) \text { e } \boldsymbol{m}_{\boldsymbol{i}}(\boldsymbol{k}+1)=\boldsymbol{m}_{\boldsymbol{i}}+\boldsymbol{v}_{\boldsymbol{i}}(\boldsymbol{k}+1) \Delta \boldsymbol{t} \\
& \operatorname{com} \boldsymbol{\phi}\left(\boldsymbol{a}_{l o c}, \boldsymbol{a}_{g l o b}\right)=\frac{2}{2-\boldsymbol{a}_{l o c}-\boldsymbol{a}_{g l o b}-\sqrt{\left(\boldsymbol{a}_{l o c}+\boldsymbol{a}_{g l o b}\right)^{2}-4\left(\boldsymbol{a}_{l o c}+\boldsymbol{a}_{g l o b}\right)}}
\end{aligned}
$$

onde, $\omega$ é a inércia, $a_{l o c}$ e $a_{g l o b}$ são as acelerações local e global, respectivamente, $a_{1}$ e $a_{2}$ são números randômicos entre 0 e 1 , e $\Delta t=1$. Cada componente do vetor velocidade é limitado a um valor máximo $v_{\max , j}$, definido por (i.e. [6]), da forma

$$
\boldsymbol{v}_{\max , j}=\boldsymbol{\alpha} \times\left(\boldsymbol{m}_{\max , j}-\boldsymbol{m}_{\min , j}\right)
$$

em que $x_{\text {max,j }}$ e $x_{\text {min, } j}$ são respectivamente os valores máximos e mínimos do espaço de busca na dimensão j, e $\alpha$ é um parâmetro tal que $\alpha \in(0,1]$. Pela descrição feita 
sobre o algoritmo PSO, é aconselhável, selecionar $a_{l o c}+a_{g l o b} \geq 4$ (i.e. [7]), isto com o intuito de garantir a convergência do método. Em geral, o algoritmo PSO básico, utilizado em problemas sem restrições, pode ser definido com os seguintes passos:

1. Inicializar o conjunto de partículas com velocidades igual a zero e posições aleatoriamente distribuídas dentro do espaço de busca;

2. Avaliar a função objetivo de cada partícula da população;

3. Atualizar a melhor posição de cada partícula e a melhor posição do bando;

4. Atualizar a posição no tempo $k+1$ baseado na posição e velocidade em $k$;

5. Repetir os processos de 2 a 4 até alcançar a condição de parada.

\section{RESULTADOS}

Dividimos os testes em duas seções: uma que aplica os métodos GA e PSO na minimização de uma função analítica, e uma outra aplicação na qual as técnicas AGFWI e PSOFWI são utilizadas na estimativa de modelos de velocidades 1D.

\subsection{GA e PSO com Função Analítica}

Para avaliar e comparar o desempenho dos métodos de inversão e GA e PSO, estudou-se inicialmente o comportamento destes na minimização da função analítica não linear multimodal convexa Rastrigin 2 (Figura 1), definida por:

$$
\boldsymbol{Q}(\boldsymbol{m})=10 \cdot \boldsymbol{n}+\sum_{\boldsymbol{i}=1}^{\boldsymbol{n}}\left[\boldsymbol{m}_{\boldsymbol{i}}^{2}-10 \cdot \cos \left(2 \cdot \pi \cdot \boldsymbol{m}_{\boldsymbol{i}}\right)\right]
$$

onde, $n$ representa a dimensão do problema, $\mathrm{m}_{\mathrm{i}}$ as variáveis da função definidas no domínio $m_{i} \in[-5,5]$ e mínimo global $Q(\boldsymbol{m})=0$ localizado em $\boldsymbol{m}=0$.

Figura 1: Função Rastring 2

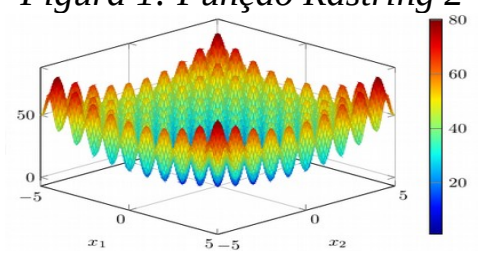

Realizou-se experimentos com diferentes configurações, fazendo $n$ variar de 2 até 40 . Para cada valor de $n$ repetimos 100 vezes o mesmo experimento, medindo o percentual de convergência com cada configuração, e tendo a equação (7) como critério de parada, onde, $\boldsymbol{m}$ é o modelo estimado, $\boldsymbol{m}^{\text {glob }}$ o mínimo teórico. Nesse experimento, dizemos que houve sucesso caso se atinja o critério $\epsilon<5.10^{-2}$, e no máximo de $10^{7}$ modelagens, dizemos que não houve sucesso.

$$
\boldsymbol{E}=\sqrt{\frac{1}{n} \sum_{i=0}^{n}\left(m_{i}-m_{i}^{g l o b}\right)^{2}}
$$

Na Figura 2 são mostrados o percentual de sucesso para cada configuração e a média com o desvio padrão da quantidade de modelagem até convergência. Esses experimentos iniciais demonstram a efetividade dos métodos GA e PSO, além de exibir a dependência da convergência como função dimensão do problema. 
Figura 2: Percentual de sucesso (esquerda) e média e desvio padrão de modelos avaliados (direita).
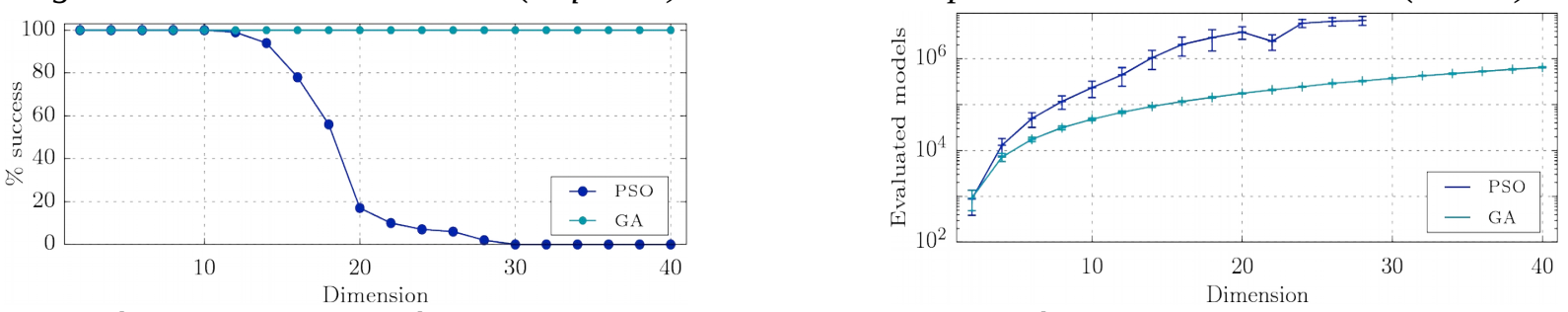

Na próxima seção será comparado o desempenho das técnicas GAFWI e PSOFWI.

\subsubsection{Resultados AGFWI e PSOFWI}

Nesta seção aplicaremos as técnicas AGFWI e PSOFWI na estimativa do perfil de velocidade sintético responsável por gerar um determinado traço sísmico. Para isso, usou-se a equação (3) na geração do dado observado $\boldsymbol{d}_{\text {obs }}$, dando como entrada o modelo $\boldsymbol{m}$ e usando AGFWI e PSOFWI, buscou-se recuperar o modelo $\boldsymbol{m}$. Realizou-se duas sequências de testes a fim de avaliar a efetividade dos métodos quando as características do problema são alteradas. Em ambos os casos mantivemos a frequência de pico $f_{\text {peak }}=11 \mathrm{~Hz}$, a taxa de amostragem na profundidade $\Delta z=10 \mathrm{~m}$ e a taxa de amostragem temporal de $\Delta t=1 \mathrm{~ms}$.

\subsubsection{AGFWI e PSOFWI: Quantidade de Parâmetros}

Como visto na figura 2, a efetividade das técnicas GA e PSO é reduzida com o aumento da quantidade de parâmetros, nesta seção isso será avaliado pelas técnicas GAFWI e PSOFWI no seis modelos da tabela 1. Para realizar a inversão, 0 espaço de busca foi mantido na faixa de $\pm 250 \mathrm{~m} / \mathrm{s}$ em relação ao modelo verdadeiro. A Figura 3 exibe os resultados dos testes. Em ambos casos, estimou-se modelos de boa qualidade e o decaimento da função objetivo mostra convergência satisfatória.

Tabela 1. Parâmetros de modelagem e inversão dos modelos 1D

\begin{tabular}{|c|c|c|c|c|c|c|}
\hline Modelo & M10 & M20 & M40 & M70 & M100 & M150 \\
\hline Camadas & 10 & 20 & 40 & 70 & 100 & 150 \\
\hline Amostras $\mathrm{z}$ & 500 & 500 & 1000 & 1600 & 2300 & 3340 \\
\hline Iterações & 100 & 300 & 300 & 400 & 700 & 1000 \\
\hline População & 200 & 200 & 300 & 600 & 800 & 1200 \\
\hline Amostra t & 2800 & 3850 & 7200 & 10570 & 16880 & 22150 \\
\hline
\end{tabular}

\subsubsection{GAFWI e PSOFWI: Domínio de Busca}

Um ponto fundamental na inversão com os métodos do tipo GA e PSO é que estes avaliam soluções em todo domínio lhe é oferecido, ou seja, a convergência influenciada pelo espaço de busca. Dessa forma, fixou-se a configuração do modelo M40, e fez-se variar o domínio de busca desde $\pm 250 \mathrm{~m} / \mathrm{s}$ até $\pm 1000 \mathrm{~m} / \mathrm{s}$, com uma população de 400 modelos e 1000 iterações. A Figura 4 mostra o comportamento da função objetivo, onde pode-se observar que para uma faixa de até $\pm 1000 \mathrm{~m} / \mathrm{s}$, existe 
Figura 3: GAFWI (azul) e PSOFWI (vermelho) para os modelos. Nos pares de figuras: função objetivo e o modelo estimado de cada teste. Em cinza os limites da faixa de busca da velocidade.

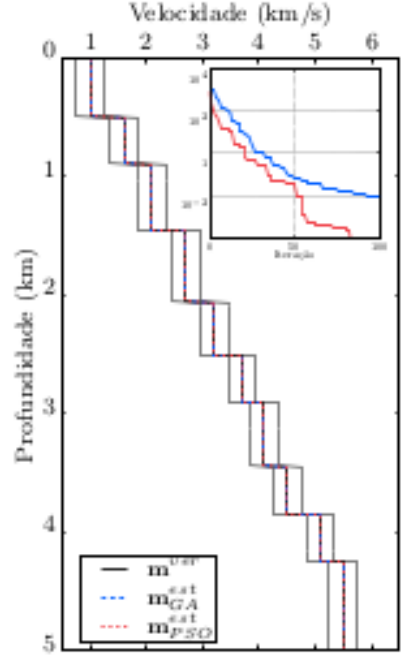

a) 10 camadas

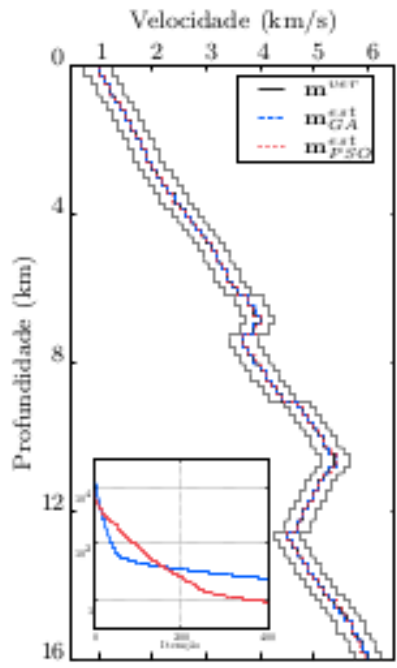

d) 70 camadas

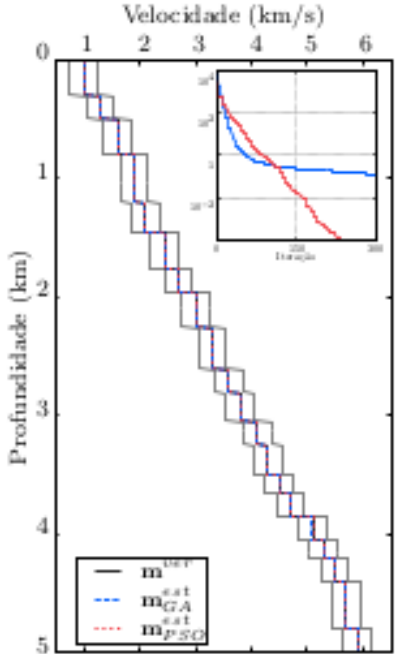

b) 20 camadas

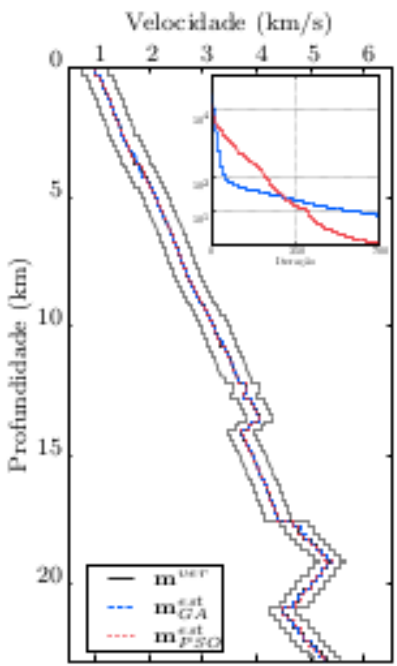

e) 100 camadas

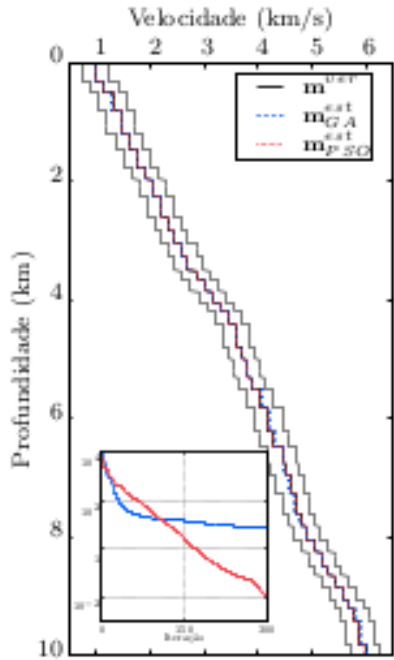

c) 40 camadas

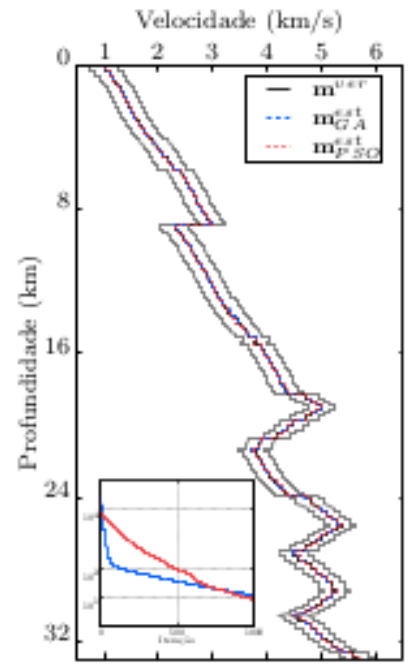

f) 150 camadas

boa similaridade entre os modelos verdadeiros e estimados.

\section{CONCLUSÃO}

Neste trabalho buscou-se avaliar a efetividade dos métodos GAFWI e PSOFWI. Inicialmente usou-se as técnicas GA e PSO para resolver o problema da função analítica, o que possibilitou concluir sobre dependência destes com a dimensão do problema. Posteriormente realizou-se experimentos semelhantes com as técnicas GAFWI e PSOFWI. No primeiro, realizamos experimentos de FWI aumentando sucessivamente a dimensão do problema, o que mostrou convergência satisfatória para o caso de até 150 parâmetros. No segundo teste foi utilizado o modelo de 40 camadas, com faixa de busca sucessivamente aumentada até atingir $\pm 1000 \mathrm{~m} / \mathrm{s}$, onde observou-se novamente também convergência satisfatória, evidenciando o sucesso dos métodos GAFWI e PSOFWI. Este segundo resultado da FWI enfatiza a natureza global dos métodos GA e PSO, pois a faixa de $\pm 1000 \mathrm{~m} / \mathrm{s}$ 
estipulada, cobre um raio de busca de cerca de $2000 \mathrm{~m} / \mathrm{s}$ que é suficiente na inversão de dados reais, em que não se conhece o modelo verdadeiro. $\mathrm{Na}$ comparação dos resultados finais do GA com PSO, pode se ver que ambas as abordagens apresentam resultados satisfatórios com pouca diferença entre si,

Figura4: Função objetivo dos GAFWI (esquerda) e PSOFWI (direita) para certas faixas de busca.
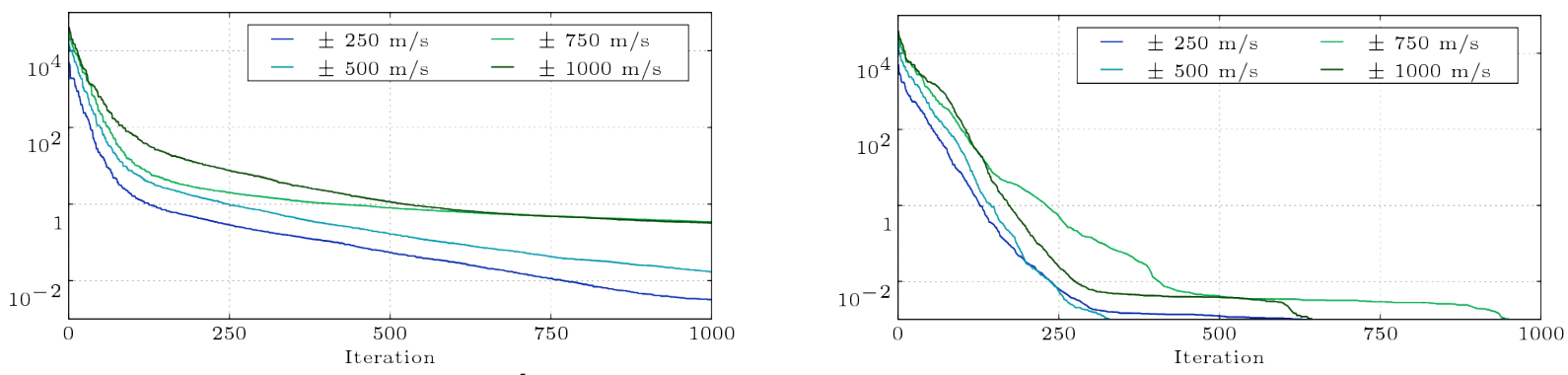

permitindo que esta estratégia seja estendida par problemas maiores, como nos casos 2D e 3D, usando tanto GAFWI como PSOFWI sem perda de efetividade.

\section{Agradecimentos}

Os autores agradecem à SHELL e ao Centro de Supercomputação do SENAI CIMATEC para Inovação Industrial pela permissão em publicar a presente pesquisa. Este projeto recebeu financiamento da Agência Nacional do Petróleo (ANP) e da Empresa Brasileira de Pesquisa e Inovação Industrial (EMBRAPII).

\section{REFERÊNCIAS}

${ }^{1}$ SANTOS, Rodrigo de S.; PORSANI, Milton J. Obtaining interval velocities using an adaptive hybrid inversion multiscale approach. In: 15th International Congress of the Brazilian Geophysical Society \& EXPOGEF, Rio de Janeiro, Brazil, 31 July-3 August 2017. Brazilian Geophysical Society, 2017. p. 1833-1838.

2SAJEVA, Angelo et al. Estimation of acoustic macro models using a genetic fullwaveform inversion: Applications to the Marmousi modelGenetic FWI for acoustic macro models. Geophysics, v. 81, n. 4, p. R173-R184, 2016.

${ }^{3}$ LINES, Larry R.et al. A recipe for stability of finite-difference wave-equation computations. Geophysics, v. 64, n. 3, p. 967-969, 1999.

${ }^{4}$ SEN, M. e P. Stoffa. Global optimization methods in geophysical inversion. Cambridge University Press, 1995.

${ }^{5}$ KENNEDY, James. Particle swarm optimization. Encyclopedia of machine learning, p. 760-766, 2010.

${ }^{6}$ ENGELBRECHT, Andries P. Computational intelligence: an introduction. John Wiley \& Sons, 2007.

${ }^{7}$ CLERC, Maurice; KENNEDY, James. The particle swarm-explosion, stability, and convergence in a multidimensional complex space. IEEE transactions on Evolutionary Computation, v. 6, n. 1, p. 58-73, 2002. 